\title{
A Mechanism for High-Frequency Oscillation in Ramjet Combustors and Afterburners
}

\author{
DON E. ROGERS ${ }^{1}$ and FRANK E. MARBLE ${ }^{2}$
}

Daniel and Florence Guggenheim Jet Propulsion Center, California Institute of Technology, Pasadena, Calif.

\begin{abstract}
An experimental investigation was made of the behavior of a small two-dimensional combustion chamber, burning a uniform mixture of air and fuel vapor under conditions of high-frequency oscillation or screech. Measurements were made of the limits of stable screech, the amplitude and frequency of pressure oscillations over a wide range of mixture ratio, inlet air temperature, and combustor flow rate. Spark schlieren photographs and high-speed motion pictures taken of the combustion process showed, in agreement with other investigations, that the high-frequency oscillation is accompanied by vortices shed periodically from the flameholder lip with the same frequency as the oscillation. The following mechanism of exciting the oscillations is suggested. A mode of transverse oscillation is excited as the result of periodic transport of combustible material, associated with the vortices, into the hot wake of the flameholder. The vortices, in turn, are generated at the flameholder lips by the fluctuating transverse velocity. When the ignition time delay lies in the proper range, the phase relationship between oseillations in transverse velocity and combustion intensity is such that the oscillation is amplified.
\end{abstract}

\section{Introduction}

$\mathbf{T}$ $\mathrm{HE}$ phenomenon of screeching combustion instability in ramjets and afterburners is characterized by its relatively high and extremely regular frequency and by the fact that the sustaining mechanism is not associated with periodic fluctuations in fuel injection or air flow rates. In addition to an intense high-frequency acoustic disturbance, screeching combustion is accompanied by marked shortening of the flame zone, severe increase in heat transfer rates, and, frequently, failure of the combustion chamber. A complete understanding and control of these high-frequency combustion instabilities constitute one of the central problems in development of high performance afterburners and ramjet burners.

Although most early discussion of the problem was confined to private communications, it is relatively clear that this unique phenomenon and some features of its mechanism were first recognized by workers in the Research Division of the United Aircraft Corporation. More recently the problem of resonant oscillations in air-breathing combustion systems has been discussed by R. Newton and J. Truman $(1)^{3}$ showing that, for several burners of different sizes, the observed frequencies of screech instability correspond to some simple mode of transverse oscillation in the burner. Although the role of transverse oscillations is certainly an important one, a comparison of frequencies does not constitute a very sensitive criterion of the mechanism since the frequencies of high order modes are rather closely spaced.

Received Sept. 15, 1955.

${ }^{1}$ Graduate Student; at present, Assistant Senior Engineer, Aerojet-General Corp. Mem. ARS.

2 Associate Professor.

? Numbers in parentheses indicate References at end of paper.
Furthermore an unstable oscillation, that is, one which is amplified by some feeding mechanism, need not correspond exactly to a normal mode. Oscillatiory frequencies of cylindric burner configurations were computed by F. K. Moore and S. H. Maslin (2) assuming that pressure sensitivity of the combustion process accounts for the excitation. The most detailed measurements that have appeared are those of W. E. Kaskan and A. E. Noreen (3) in which schlieren photographs provide considerable detail of the process. There is a similarity between the photographic studies presented by these authors and some of those discussed here. Kaskan and Noreen suggest that the exciting disturbance arises from the distortion or stretching of the flame front near the flameholder, caused by the acoustic disturbance. The influence of flame distortion was also discussed previously in this regard by P. L. Blackshear (4).

The present paper covers a set of experiments performed in a small burner during 1953, for the purpose of elucidating the high-frequency instability; they were first reported in (5). Measurements were made of screech limits, amplitude, and frequency of the oscillation; photographic studies were obtained of the combustion details. Although the experimental results are incomplete in many ways, they add considerable interesting detail to existing published work. As a result of these studies, furthermore, the authors suggest a mechanism that is essentially different from those previously set forth.

The authors are most grateful to members of the staff of the Jet Propulsion Laboratory, California Institute of Technology, for assistance in carrying out the experimental work described herein and for the use of their excellent facilities. The two schlieren photographs which appear as Figs. 13 and 14 were obtained by Calvin L. R. Barker of the Jet Propulsion Laboratory during the current extension of the experimental work.

\section{Experimental Exploration of Screeching Phenomenon}

The exploratory investigation of screeching combustion was carried out in a small combustion chamber of rectangular cross section in order that the observed phenomena would be of nearly two-dimensional character. Mixtures of air and vaporized fuel entered a large plenum chamber to a converging nozzle having a contraction ratio of $28 / 1$, to the combustion chamber, and then were discharged at atmospheric pressure. A photograph of the nozzle and working section is shown in Fig. 1 . Air was supplied at a sufficiently high pressure that the flow rate could be accurately controlled by means of an adjustable sonic throat. Mixture temperature in the working section was controlled to within $\pm 5 \mathrm{~F}$ by passing the air through a conventional shell-andtube heat exchanger located far upstream of the settling chamber. A gasoline type fuel was injected into the airstream about 40 feet ahead of the plenum chamber so that a completely uniform mixture of fuel vapor and air was obtained at the working section under all conditions. 
The plenum chamber contained six sereens, of 150 mesh each, located so as to reduce large-scale disturbances and to provide general control of the turbulence level. The resulting flow entering the combustion chamber had a uniform velocity profile, except within boundary layer regions of normal thickness, and a turbulence of low but undetermined level.

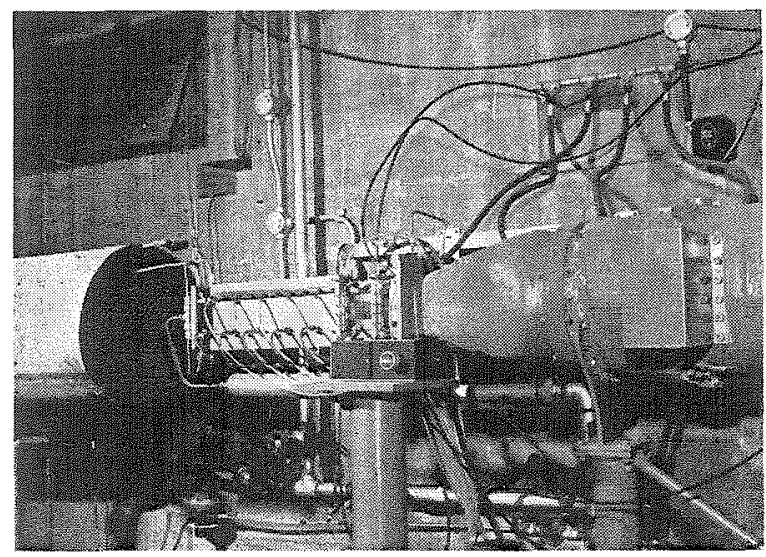

Fig. 1 View of the plenum chamber and nozzle

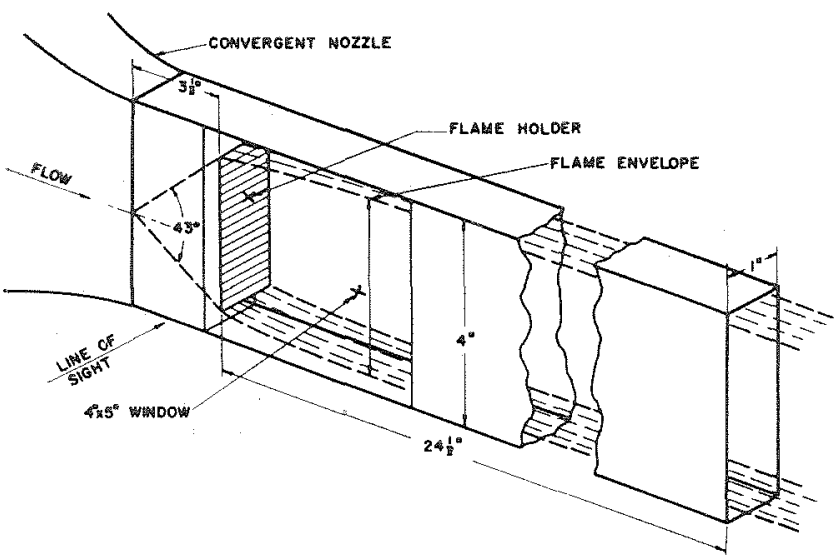

Fig. 2 Schematic diagram of the experimental combustion chamber

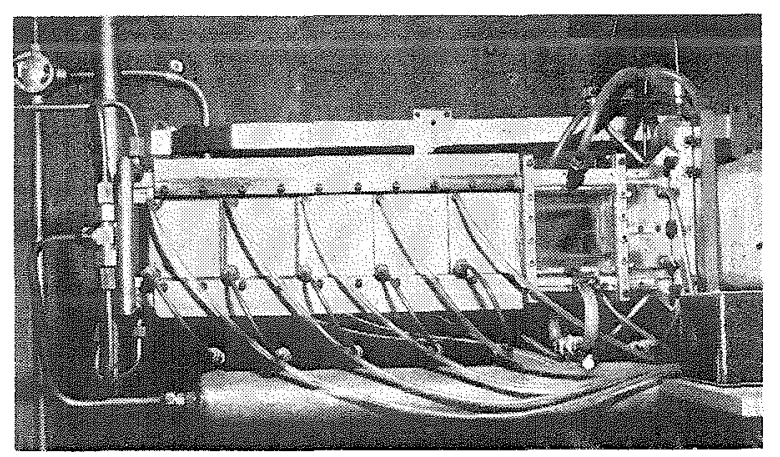

Fig. 3 Side view of the combustion chamber

A schematic diagram of the combustion chamber and flameholder is shown in Fig. 2. The combustor was of $1 \mathrm{in}$. by 4 in. rectangular cross section and extended $24 \frac{1}{2}$ in. in length beyond the end of the wedge-shaped flameholder. The apex of the fameholder was located $1 \frac{1}{2}$ in. downstream of the chamber inlet. Vycor glass windows permitted a feld of view consisting of the region $4 \mathrm{in}$. by $5 \mathrm{in}$. immediately downstream of the flameholder. The chamber was fabricated of stainless steel to alleviate the corrosion problem arising from cooling water in the walls. The side walls of the burner were constructed of plate segments, Fig. 3, each of which was separately cooled and sealed. Since the plates were each 4 in. long in the axial direction, the design per- mitted variation of burner length in increments of 4 in

The flameholder used throughout the investigation was a solid wedge with apex angle of $43 \mathrm{deg}$ and an axial length of $3 \frac{1}{2}$ in. It was constructed of stainless steel and was equipped with an independent water-cooling circuit. Ignition was accomplished by inserting an electrode into the combustion chamber and allowing a spark to jump to the downstream side of the flameholder. This probe could be retracted after ignition to allow smooth unobstructed gas flow. A complete description of equipment details and experimental procedures is given in (5).

\section{Frequency and Amplitude Measurements}

It was observed during the present experiments, as well as in the work of previous investigators, that the simplest and least destructive technique for entering the regions of screeching combustion was to increase the fuel-air ratio of the mixture ratio while holding the airflow rate and other freestream conditions constant. The most direct indication of screech is the total pressure loss across the combustion chamber. Roughly speaking, the total pressure loss is a measure of the heat release that takes place within the combustion chamber; as this heat release increases, the momentum pressure loss across the combustor increases. Since, during screeching operation. the flame zone is observed to contract substantially, a greater portion of the burning process takes place within the chamber so that the onset of screech is accompanied by an increase in pressure loss.

A typical diagram illustrating the transition into an out of screeching operation is shown in Fig. 4 where the dimensionless pressure loss across the combustion chamber is given for a wide range of fuel-air mixture ratios. The total pressure loss is reduced to a dimensionless form through division by the absolute stagnation pressure at the combustion chamber inlet; the mixture strength is presented as the equivalence ratio, that is, the quotient of actual fuel-air ratio to the fuelair ratio at stoichiometric. For the free stream speed or air mass flow shown, the lean blowoff limit of the flameholder occurred at an equivalence ratio of about 0.72 . As the equivalence ratio was increased to about 0.85 , the combustor entered screeching operation as indicated by the substantial increase in pressure drop. Screeching operation continued at greater values of mixture ratio until the rich instability limit was reached. Here the combustion process may be described as rough, that is, exhibiting a series of random pulsations or explosions of considerable violence. As the equivalence ratio was reduced, the pressure loss retraced its previous trend until it reached the region of transition between screeching and normal operation. Here it remained in sereech until the mixture ratio was considerably lower than that required for the transition into screech. Although such a hysteresis effect may be associated with a pulsation involving finite amplitudes, the effect shown here is probably associated with heat transfer. When the burner is operating in the sereeching condition, portions of the

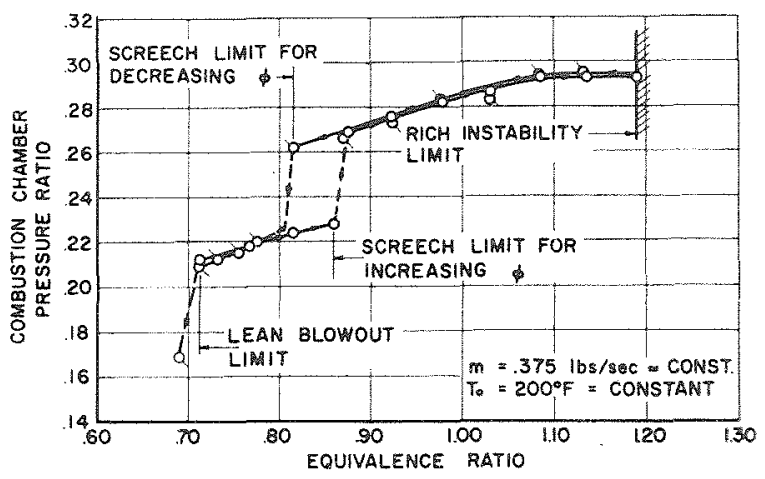

Fig. 4 Variation of combustion chamber inlet total pressure with equivalence ratio 


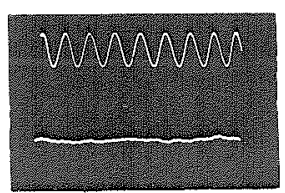

$\emptyset=.731$

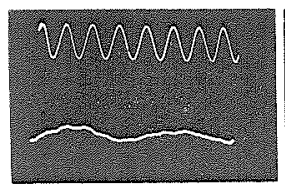

$\emptyset=.890$

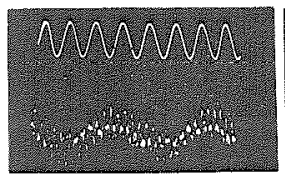

$0=.966$
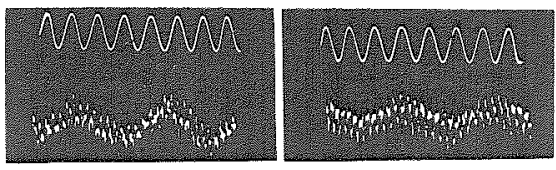

$T_{0}=250^{\circ} \mathrm{F}$

$\mathrm{m}=.300 \frac{1 \mathrm{bs}}{\mathrm{sec}}$

$\theta=1.125$

$\phi=1.176$

Fig. 5 Typical pressure fluctuation records for a range of equivalence ratios

chamber heat up appreciably above the temperatures encountered during steady operation. Inasmuch as it will appear that the screech limit occurs at leaner mixture ratios when the gas temperature is higher, it is reasonable to suppose that heating of the chamber itself would affect it in a similar manner. This accounts for the hysteresis appearing in Fig. 4.

Frequency and amplitude of the oseillations are of particular significance and these were measured with Photocon capacitance-type gages of 100 psi range and a natural frequency of 75,000 cps. The output of each gage was observed on a Dumont dual beam oscilloscope and recorded photographically. The gages were water cooled, mounted flush with the inside surface of the wall, and could be installed at a variety of positions on the duct. A typical set of response patterns for various mixture ratios is shown in Fig. 5 where the gage is located on the center line of the duct, $11 \mathrm{in}$. downstream from the flameholder face. In each diagram the lower curve represents the trace of the pressure oscillation while the upper curve is the timing frequency of $1000 \mathrm{cps}$. The onset of screeching operation is clearly delineated by the increase in amplitude of the pressure oscillation. The pulsations clearly consist of two distinct frequencies, a low-frequency oscillation of about $285 \mathrm{cps}$ and the high-frequency oscillation, characteristically associated with screech, of about 3800 cps. The lowfrequency oscillation corresponds roughly to the longitudinal mode of the chamber resonating as if it were closed at the flameholder, while the high-frequency oscillation corresponds roughly to the fundamental (antisymmetric) transverse mode across the 4 -in. dimension of the duct. The high-frequency component is of principal interest. Because the gage was located on the center line of the duct for the photographs of Fig. 5, the relative magnitude of the high-frequency component is shown considerably too small. The significance of pressure measurement location has since been discussed in (6).

The frequency of the screeching oscillation is quite insensi-

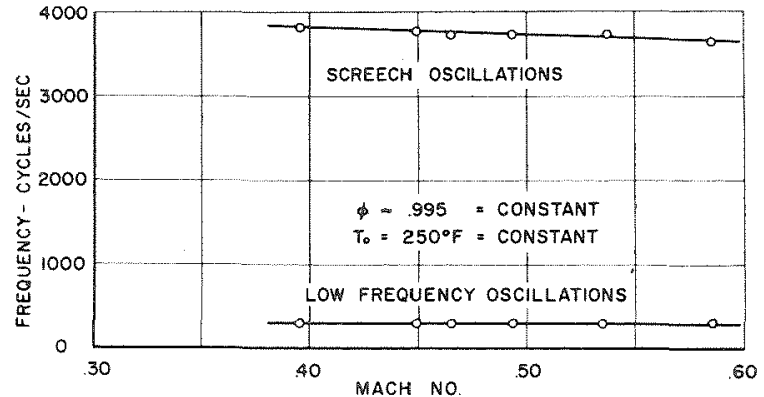

Fig. 6 Variation of frequency with the average Mach number at the flameholder lip

tive to changes in mixture ratio and, as indicated in Fig. 6 , decreases only in a minor way as the airflow rate through the combustion chamber is increased. The Mach number employed as the ordinate in Fig. 6 is an average one based on the total mass flow, unblocked cross-sectional area for flow at the flameholder lip, and the free stream conditions. This is used as a convenient indication of the Mach number near the flameholder lip, which is the significant value in the present problem. Fig. 6 also shows that the frequency of longitudinal oscillation is completely unaffected by the changes in flow rate.

Measurements of fluctuating pressures permitted a rough determination of amplitude of the pressure oscillations and the manner in which it varied with mixture ratio and average Mach number. The amplitudes of high- and low-frequency components of oscillation are shown in Fig. 7 where the ratio of peak-to-peak pressure difference to the stagnation pressure at the combustion chamber inlet is used as the ordinate. For these measurements the pressure pickup was located on the top wall of the combustion chamber $1 / 4 \mathrm{in}$. downstream of the flameholder lip. The pressure amplitudes shown are therefore approximately those of importance in screech excitation. The onset of screech at an equivalence ratio of about 0.90 is indicated by a large increase in amplitude of pressure oscillation. ${ }^{4}$ Below this value the amplitude of high-frequency oscillation is barely perceptible but, after its initial jump, increases rather slowly with mixture ratio. The low-frequency longitudinal component is present, as indicated in Fig. 5, even below the screech limit, although its amplitude also doubles when screech is encountered. It appears likely that the longitudinal oscillation is excited to moderate amplitude by any disturbance in the chamber such as random pulses associated with rough burning. The abrupt increase at screech indicates some coupling with the high-frequency component or with the generating mechanism, but it appears that, at least in the present case, the exeiting mechanism is associated with the high-frequency component. Changes in length of the combustion chamber changed the frequency and amplitude of the low-frequency component but had negligible effect upon the screech component. Thus it seems clear that the excitation took place in the high-frequency component and the screech phenomenon is relatively independent of the longitudinal oscillation.

Although the limited results available are not conclusive on this point, it was observed that the screech amplitude tended to decrease as the average lip Mach number increased and that high values of the Mach number inhibited screech. At average Mach number values of the order of 0.90 the combustor screeched only intermittently. As the Mach number was further increased it was not possible to initiate screech, and the combustor operated smoothly over the entire range of mixture ratios.

4 These metsurements were made in a chamber with cooling properties different from the chamber employed during other measurements. This feature accounts for the difference in lean screech limit. 


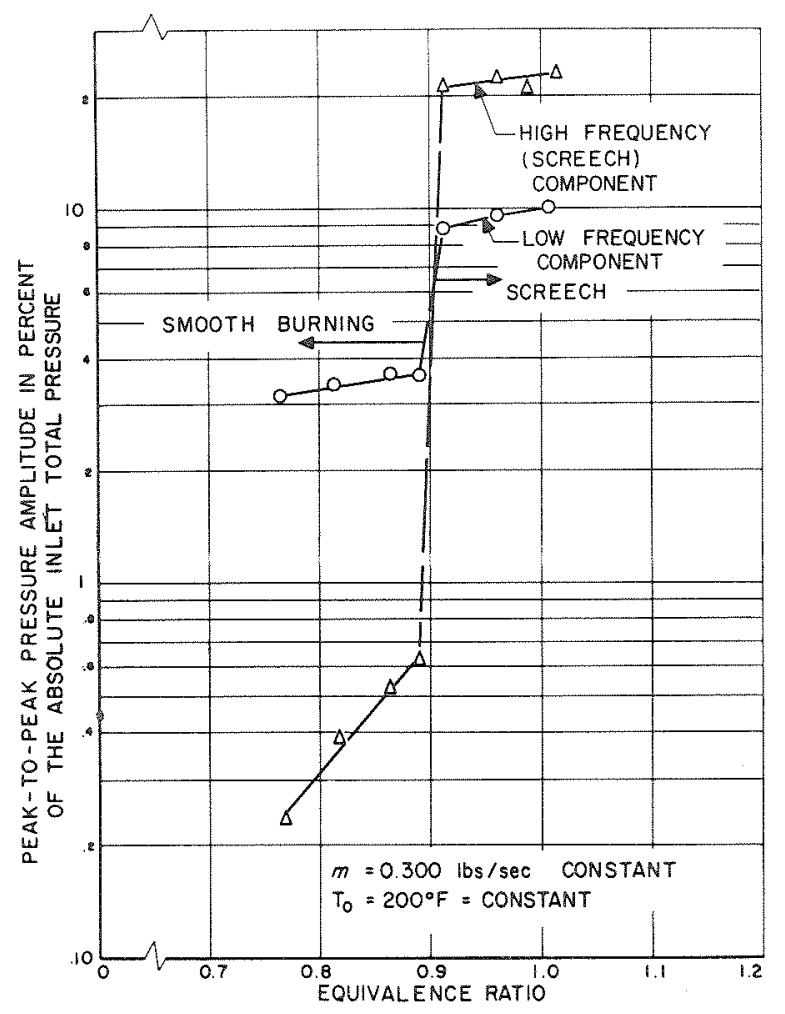

Fig. 7 Amplitude of pressure fluctuations for a range of equivalence ratios

\section{Limits of Stable Operation}

As indicated previously, there are mixture ratio limits within which the combustor must operate in order that stable screech be observed. Of particular interest is the lean screech limit because this limit appears to be associated directly with the exciting mechanism while the rich limit is not. This lean limit is rather insensitive, Fig. 8, to variations in lip Mach number; except for a substantial change at very low flow rates, the lean limit remains at an equivalence ratio of about 0.85 . The amplitude of oscillation decreased as the

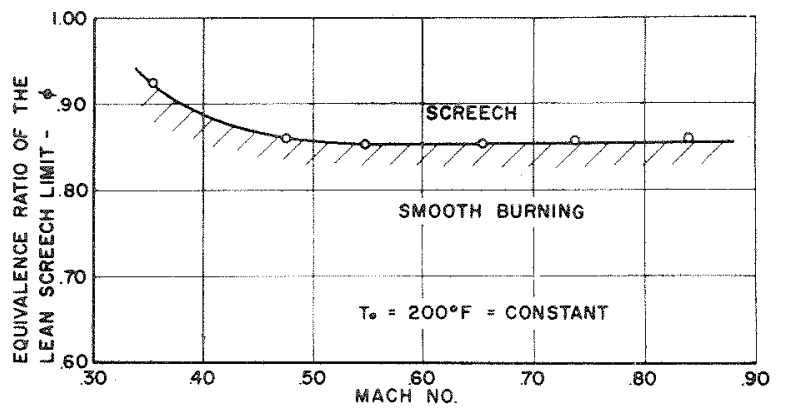

Fig. 8 Variation of the lean screech limit with average Mach number

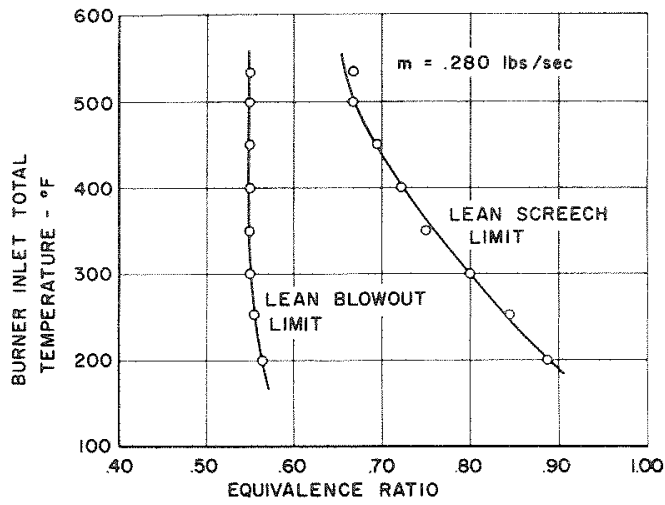

Fig. 9 Variation of lean screech limit with inlet total temperature
Mach number increased but, below an average Mach number of 0.9 , this has no influence upon the lean limit.

Since combustion processes are notoriously sensitive to initial temperature of the mixture, it is to be expected that the lean screech limit will be significantly influenced by changes in temperature of the mixture entering the combustion chamber. This conjecture is confirmed by the observed strong decrease in screech limit, Fig. 9, as the burner inlet temperature is increased. The lean blowoff limit of the flameholder is also shown and clearly as the inlet temperature increases above $500 \mathrm{~F}$, the screech limit very nearly reaches the lean blow off limit, indicating that, for this inlet temperature, the burner will screech over its entire range of useful operation.

\section{Optical Study of the Screech Mechanism}

The most direct manner of obtaining detailed information concerning the mechanism of screeching combustion is through photographic investigation. Photographs of the region near the flameholder show that the combustion zone in the mixing region downstream of each lip is normal, Fig. 10, during smooth operation but takes on a scalloped appearance, Fig. 11, during screeching operation. Spark schlieren photographs of the process reveal considerably more detail of the flow. These optical studies were conducted using a single-pass, double-mirror schlieren system of conventional arrangement. The spark schlieren pictures were obtained using a high-intensity spark source of 2 to 3 microsec duration. The high-speed motion pictures were obtained with a Western Electric Fastax camera using a General Electric mercury vapor lamp for illumination.

A spark schlieren picture of smooth combustion is shown in Fig. 12. As described in detail in (7), the boundary layer from the bluff body separates, spreads through turbulent mixing into the hot zone of recirculating combustion products, and ignites before the end of the recirculating zone is reached, thereby producing a propagating flame. A comparable photograph of sereeching combustion, Fig. 13, shows the profound modification of the flow field that accompanies the

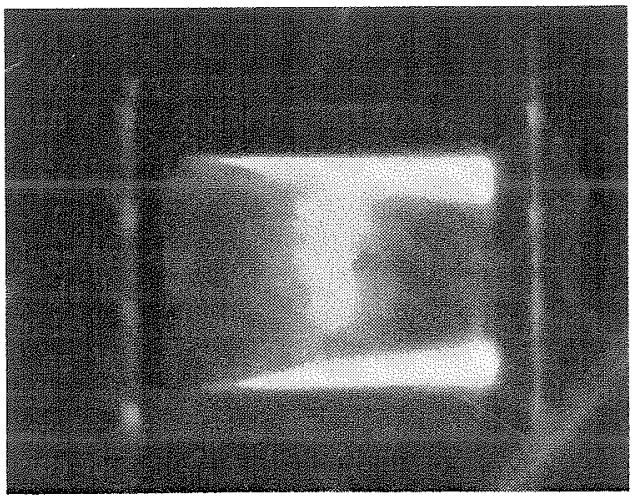

Fig. 10 Direct photograph of smooth combustion

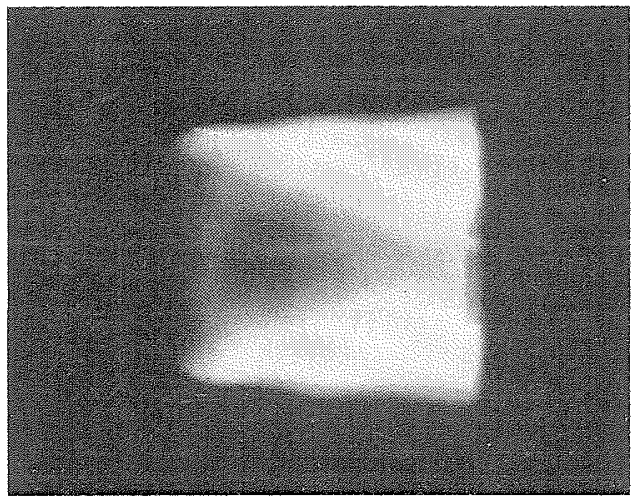

Fig. 11 Direct photograph of screeching combustion 


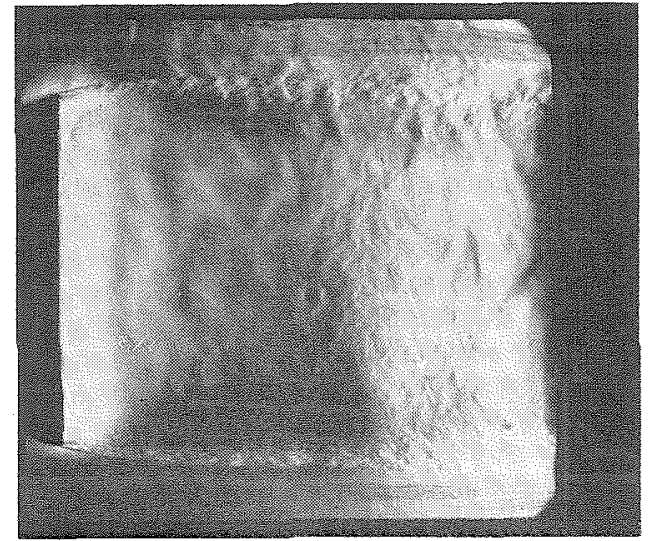

Fig. 12 Spark-schlieren photograph of smooth combustion

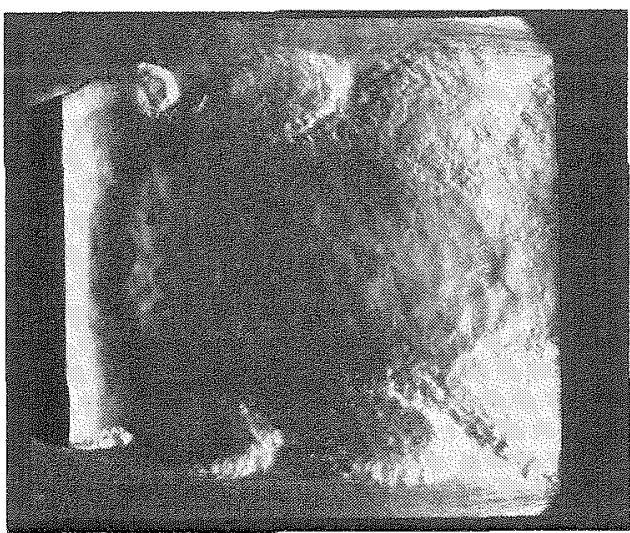

Fig. 13 Spark-schlieren photograph of screeching combustion

pressure fluctuations. The transverse fluctuations across the combustion chamber are associated with the formation of vortices which are, in turn, shed alternately from the lips of the flameholder. In this photograph one vortex is in the process of formation on the lower lip while a fully developed vortex has drifted downstream from the upper lip; the transverse (upward) velocity, existing at the time of the photograph, has moved both of them off center toward the upper part of the photograph. Vortices from previous cycles, burned and partially decayed, appear downstream in the wake.

Now if these vortices are associated with the highfrequency transverse oscillation, they must be shed with the same frequency. To obtain this information in particular and a more complete picture of the shedding process in general, high-speed schlieren motion pictures were taken of the screeching combustion. The maximum camera speed of about 7000 frames per sec was insufficient to obtain detailed history of one vortex. However, by photographing the process while the camera frame frequency was accelerating through the frequency of vortex shedding, successive positions of successive vortices could be traced, thereby giving the effect of slowing down the process. This also provided, through the timing marks on the film, an accurate means for obtaining the frequency of vortex shedding. Two sequences of pictures are shown in Fig. 14. Figs. 15 and 16 compare the vortex shedding frequencies thus obtained with the screech frequencies from pressure measurement for a range of lip Mach numbers and equivalence ratios. The agreement, well within accuracy of the measurement, leaves little doubt that the two phenomena are associated and that the vortex wake is in no way connected with the Kármán vortex street formed in the wakes of bluff bodies in a certain Reynolds number range. It seems apparent, in fact, that the vortices are formed as a result of the transverse velocity associated with the sloshing oscillation. Referring for convenience to Fig. 13 , the upward velocity associated with a particular phase of

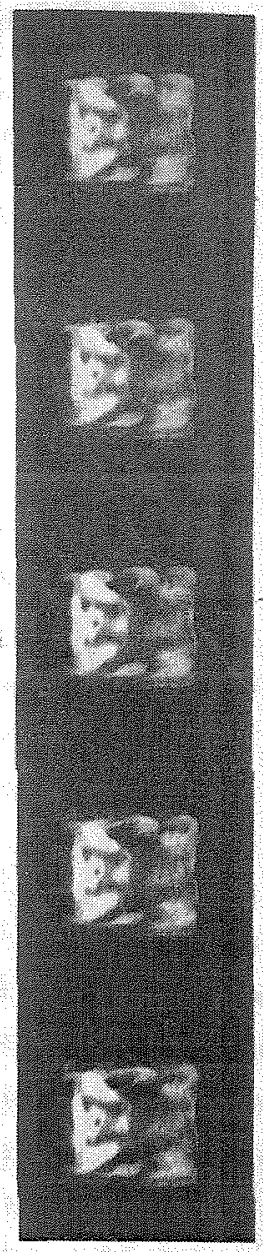

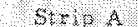

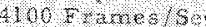
0. 1.180

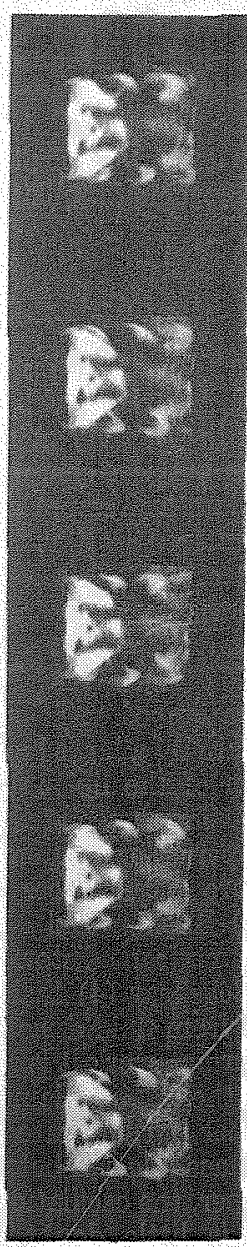

$\sin 10$

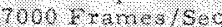
$6=1,100$
Fig. 14 Typical high-speed schlieren motion pictures of screeching combustion

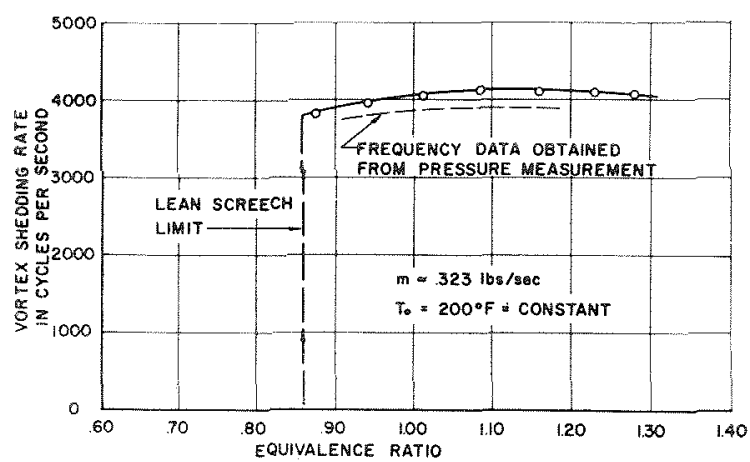

Fig. 15 Variations of vortex shedding rate with equivalence ratio

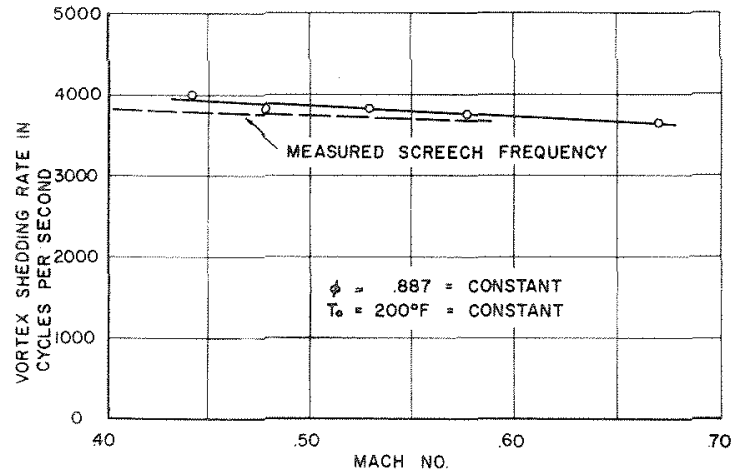

Fig. 16 Variation of vortex shedding rate with Mach number 
the pressure oscillation induces a flow about the lower lip conducive to the formation of a vortex. In a later phase of the motion the transverse velocity is reversed and a similar vortex is formed at the upper lip. Thus the frequency of vortex formation at each lip will be equal to the frequency of the screeching oscillation. This corresponds to the observation.

\section{Mechanism of Screeching Combustion}

At least two proposals have been made concerning mechanisms by which the high-frequency oscillations may be excited. In their analytic study of the predominant modes of combustion chamber pulsation, Moore and Maslen (2) postulate a pressure dependent burning rate similar to that encountered in liquid propellant rocket motors. Although hydrocarbon mass combustion rates are dependent upon the pressure they are equally or more sensitive to changes in the temperature. The experiments reported here as well as those discussed in (3) do not indicate pressure fluctuation of sufficient magnitude to raise the local temperature by a large factor. Since the gas immediately downstream of the lip may be subjected to violent temperature fluctuations associated with the mixing process, it seems likely that periodic temperature fluctuations would overshadow pressure disturbances in exciting variations of burning rate. In (3) Kaskan and Noreen consider a mechanism involving the flame front distortion and employ the original suggestion of Blackshear (4) that the fluctuations in burning rate may resilt from fluid dynamic stretching of the flame front. In the former work it is reasoned that the vortex formation extends the existing flame front and thus produces, periodically, a higher burning rate and a resulting pressure pulse. When the phase relation between vortex formation and combustion is proper, the oscillation will be amplified or sustained. Now there is little doubt that, under proper conditions, such changes in flame area may excite pressure fluctuations, but under the circumstances of combustion in a region of high shear and intense turbulence, such as exists in the separated boundary layer, the concept is somewhat vague. The rate of combustion increases linearly with the flame front area but much more rapidly due to increase in ambient temperature. Since the phenomenon is so intense it seems that a more vigorous means of excitation dominates the picture associated with mixing between combustible gas and hot products of combustion in the wake. On the basis of existing experiments on high-frequency oscillations in combustion chambers and the present knowledge of processes involved in flame stabilization on bluff bodies, it is suggested that the following mechanism excites and sustains the oscillations.

Under conditions of normal smooth operation the combustible gas passes about the bluff body, separates from the surface, and mixes with the hot recirculating wake in a thin turbulent shear zone. As shown in (7), if the wake is sufficiently long and the recirculating zone sufficiently hot, the fresh gas will be ignited and a propagating flame established. During high-frequency oscillation, fluctuating pressure, temperature, and velocity fields are superimposed upon this steady field; in particular an oscillating transverse (or sloshing) velocity field is added to the existing field for steady combustion. As the flow angle with respect to a flameholder lip is increased by this transverse velocity, a vortex forms, Fig. 17, similar to the starting vortex formed when an airfoil enters a cross velocity field or gust. The transverse velocity which formed the vortex helps transport it into the hot recirculating wake zone of the flameholder. Since the vortex consists principally of unburned gas from the boundary layer of the bluff body, this transport process carries a large amount of combustible material into the hot zone. The shear layer which formed the vortex is strongly turbulent so that this combustible material mixes with the hot wake gas and, after an appropriate length of time, ignites and burns.
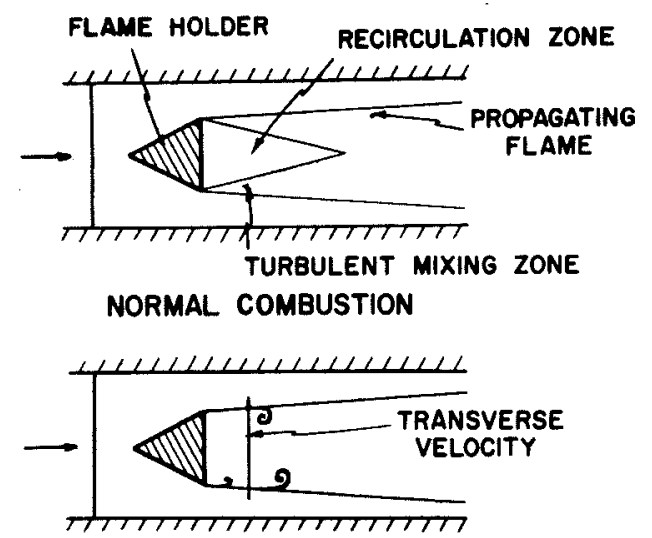

HIGH FREQUENCY OSCILLATION

Fig. 17 Vortex formation associated with transverse velocity field

The combustion of this large amount of material generates a pressure wave which in the present circumstances, since the vortex is off center in the duct, excites the antisymmetric modes of the duct. To support an oscillation of large amplitude, this exciting process must (a) occur in the proper phase of the cycle to provide amplification, and (b) be of sufficient magnitude to overcome the various damping influences present.

\section{The Ignition Time Delay}

The combustion oscillation lags the oscillations in transverse velocity because of the time required to form the vortex as well as the time required for mixing and ignition of the fresh gas associated with the vortex. The fluctuations in transverse velocity and combustion rate must bear a certain phase relation in order that energy may be fed into the oscillating motion. The classical criterion for reinforcement of oscillations by heat release is that the heat be released when the pressure at the point of heat release is near its maximum value. Thus to excite the fundamental transverse mode of oscillation in the present configuration the heat should be released as the gas mass starts toward the center of the duct from the side where the combustion pulse took place. This condition is fulfilled either when both vortex formation and burning occur early in the return cycle of gas motion, or when combustion takes place an integral number of periods after vortex formation where the transverse gas motion is again the same. Some information on the ignition time delay of such systems is available from flame blowoff experiments with bluff bodies. The flame stabilization process on bluff bodies involves also an ignition delay accompanied by a mixing process; if the time required for the combustible mixture to flow past the hot recirculation zone is less than that required to mix and ignite the combustible, then the flame will not propagate into the combustible stream and is said to blow off from the bluff body. Observations of blowoff velocity during normal combustion permit rather straightforward estimates of this time delay (8), as about $0.00028 \mathrm{sec}$ at stoichiometric mixture, inlet gas temperature of $610 \mathrm{R}$, and atmospheric pressure. Now the period of the oscillation observed in the present burner is about $0.00026 \mathrm{sec}$. Therefore it is clear that the combustion pulse does not occur directly after the vortex is formed but with a delay of about one complete period as indicated in Fig. 18. From flame stabilization experiments of (7) and (8) it appears that the ignition time delay is characterized by the fuel, increases strongly as the mixture varies from that giving maximum flame temperature, and decreases strongly as the temperature of the combustible mixture is increased. From this viewpoint, the screeching oscillation does not occur at sufficiently lean mixtures because the ignition delay increases so markedly as the mixture drops below stoichiometric that the phase of 


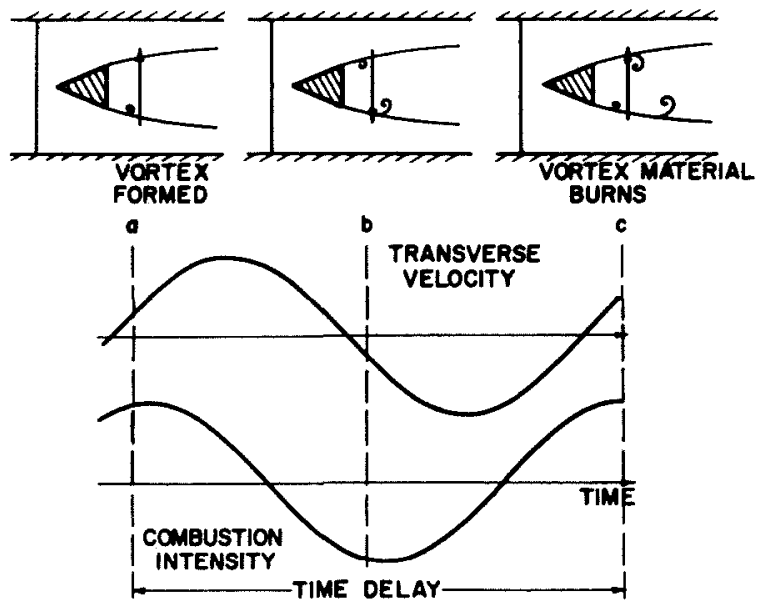

Fig. 18 Time delay required to excite transverse oscillations

excitation is improper for amplifying the oscillation. Furthermore, raising the mixture temperature tends to decrease the ignition time lag at a given mixture ratio and hence, as shown in Fig. 9, permits stable screech at leaner mixtures.

It must be emphasized that ignition after a delay of about one period need not be common to all combustion chambers. For a large chamber where the period of the fundamental transverse period is long compared with the ignition time delay of 0.00028 sec, the ignition may take place during the same cycle that manufactured the vortex. This probably occurs when transverse or radial oscillations of the order of $1000 \mathrm{cps}$ are excited in large burners. It might also be inquired whether screech should be possible when the time delay is of several periods' duration. There seems to be no observation indicating such behavior, but neither has any systematic investigation of that point been pursued. With the mechanism proposed, however, excitation after several periods' time delay seems rather improbable for two reasons. First, if the delay between vortex formation and combustion is very long, both the vortex and the mixing region between stream and wake become quite diffuse in comparison with the amplitude of transverse gas motion. The original vortex material would then have associated with comparable amounts of fresh gas and combustion products so that local high-intensity combustion should be suppressed. Second, a delay of several periods situates the vortex a considerable distance downstream of the flameholder when combustion takes place. This configuration is less advantageous for exciting transverse oscillation than that of combustion taking place quite near the flameholder face.

\section{Damping Influences}

Although viscous damping, surface absorption, etc., certainly play roles in the screeching phenomenon, the principal damping effect of the present experiments is the transport of fresh undisturbed gas into the burner region. This damping force arises physically from the fact that the energy associated with the transverse oscillation is continually being transported out of the chamber while fresh gas is entering in which the oscillation must be generated. The damping force is proportional to the mass flow rate through the combustor and its phase is always such as to decrease the amplitude of oscillation. A high free stream velocity may be expected to have a further tendency to damp the motion because it transports the vortex farther downstream within one cycle than does a low stream velocity. Consequently, since the hot recirculating zone is of fixed length, the vortex may eventually be transported out of the main portion of the wake before mixing and combustion may be effected. Finally the influence of the local Mach number at the flameholder lip may be considered. As the Mach number approaches and locally exceeds unity, the pressure pulse originating at the burning vortex can influence the flow pattern only within the Mach wedge and hence is incapable of generating another vortex. Consequently, in agreement with observation, screeching combustion cannot be supported as the lip Mach number exceeds unity.

\section{Concluding Remarks}

The experiments performed and the mechanism suggested pertain, in detail, only to a very simple combustion chamber with essentially two-dimensional flow. Although the mechanism probably does not account for all combustion chamber oscillations of high frequency, the ideas may be extended fruitfully to a wide variety of geometric conditions. In the first place, only the fundamental transverse mode of oscillation was observed. It is clear that, for a given fluid dynamic and ignition delay in burning the vortex, that mode will be observed for which this phase lag produces the greatest divergence. For example, increasing the chamber size would result in selection of higher modes such that the frequencies are of the same general order as that observed in the small chamber. However, when the burner size becomes so large that the period of some mode is long enough to permit combustion during the same cycle that produced the vortex, distinctly lower frequencies should be observed. In a burner of axially symmetric geometry, two patterns of screech appear possible. The vortices may be shed either periodically as discrete rings or continuously as a helical vortex line.

Simple methods for alleviating screech also suggest themselves; the general aim would be to arrange the burner so that an induced mode of oscillation would not manufacture vortices in the critical region. One obvious technique is to prevent reflection at the burner shell or to scatter the reflected wave so that the induced velocities near the flameholder are small. Another technique would be to disperse the flameholding element (axially and radially) in such a fashion that pressure waves are not reflected with great strength in the neighborhood of any flameholder. Such preventative means would be of particular importance in afterburners where the burner inlet temperature is so high that the unstable region would cover the entire range of useful mixture ratios.

\section{References}

1 Newton, R. T., and Truman, J. C., "An Approach to the Problem of Screech in Ducted Burners," Transactions, The American Society of Mechanical Engineers (to be published).

2 Moore, Franklin K., and Maslen, Stephen K., "Transverse Oscillations in a Cylindrical Combustion Chamber," NACA Technical Note no. 3152 , Oct. 1954.

3 Kaskan, W. E., and Noreen, A. E., "High Frequency Oscillations of a Flame Held by a Bluff Body," presented at Annual Meeting of the ASME, New York, N. Y., Nov. 28, 1954.

4 Blackshear, P. L., "Driving Standing Waves by Heat Addition," NACA Technical Note no. 2772, 1952.

5 Rogers, Don E., "An Experimental Investigation of High Frequency Combustion Instability in a Fuel Air Combustor," Thesis for the degree of Aeronautical Engineer, California Institute of Technology, May 1954.

6 Blackshear, P. L., Rayle, W. D., and Tower, L. K., "Study of Screeching Combustion in a 6-inch Simulated Afterburner," NACA Technical Note no. 3567, Oct. 1955.

7 Zukoski, E. E., and Marble, Frank E., "The Role of Wake Transition in the Process of Flame Stabilization on Bluff Bodies," Combustion Researches and Reviews, 1955, The Advisory Group for Aeronautical Research and Development, NATO, Butterworths Scientific Publications, London, 1955.

8 Zukoski, E. E., and Marble, Frank E., "Experiments Concerning the Mechanism of Flame Stabilization in the Wakes of Bluff Bodies," Proceedings of the Symposium on Gas Dynamics, Northwestern University, August 22-24, 1955. 